\title{
Parental unemployment associated with the lack of the effectiveness of a children obesity prevention program: Results from the IDEFICS study
}

\author{
Alelí M. Ayala-Marín $^{1,2}$, Pilar De Miguel-Etayo ${ }^{1,3}$, Javier Santabárbara ${ }^{4,5}$, Isabel Iguacel ${ }^{1,3}$, \\ Wolfgang Ahrens, ${ }^{6,7}$, Stefaan De Henauw ${ }^{8}$, Lauren Lissner ${ }^{9}$, Eszter Molnár Kurdine ${ }^{10}$, \\ Reisch Lucia ${ }^{11}$, Paola Russo ${ }^{12}$, Michael Tornaritis ${ }^{13}$, Toomas Veidebaum ${ }^{14}$ and Luis \\ A. Moreno ${ }^{1,15}$ \\ ${ }^{1}$ Growth, Exercise, Nutrition and Development (GENUD) Research Group, Universidad de Zaragoza, Zaragoza, Spain, \\ ${ }^{2}$ Instituto de Investigación Sanitaria Aragón (IIS Aragón), Universidad de Zaragoza, Zaragoza, Spain, \\ ${ }^{3}$ Centro de Investigación Biomédica en Red de Fisiopatología de la Obesidad y Nutrición (CIBERObn), Instituto de \\ Salud Carlos III, Ministerio de Ciencia, Innovación y Universidades, Madrid, Spain, \\ ${ }^{4}$ Centro de Investigación Biomédica en Red de Salud Mental (CIBERSAM), Instituto de Salud Carlos III, Ministerio \\ de Ciencia, Innovación y Universidades, Madrid, Spain, \\ ${ }^{5}$ Department of Microbiology, Preventive Medicine and Public Health, University of Zaragoza, Zaragoza, Spain, \\ ${ }^{6}$ Department of Epidemiological Methods and Etiologic Research, Leibniz Institute for Prevention Research and \\ Epidemiology-BIPS, Bremen, Germany, \\ ${ }^{7}$ Institute of Statistics, Faculty of Mathematics and Computer Science, University Bremen, Bremen, Germany, \\ ${ }^{8}$ Department of Public Health, Faculty of Medicine and Health Sciences, Ghent, Belgium, \\ ${ }^{9}$ Section for Epidemiology and Social Medicine (EPSO), Sahlgrenska Academy, University of Gothenburg, \\ Gothenburg, Sweden, \\ ${ }^{10}$ Department of Languages for Specific Purposes, Medical School, University of Pécs, Pécs, Hungary, \\ ${ }^{11}$ Department of Intercultural Communication and Management, Centre for Corporate Social Responsibility, \\ Copenhagen Business School, Copenhagen, Denmark, \\ ${ }^{12}$ Institute of Food Sciences, CNR, Avellino, Italy, \\ ${ }^{13}$ Research and Education Institute of Child Health, Nicosia, Cyprus, \\ ${ }^{14}$ Department of Chronic Diseases, National Institute for Health Development, Tallin, Estonia and \\ ${ }^{15}$ Departamento de Fisiatría y Enfermería, Universidad de Zaragoza, Zaragoza, Spain
}

Abstract

The relationship between social vulnerabilities and the effectiveness of behavioral interventions to prevent obesity in children is poorly understood. Therefore, the objective of this study is to evaluate the association between parental employment and the effectiveness of IDEFICS, a multilevel behavioral intervention aiming to prevent obesity among children (2 to 9.9 years old) in eight European countries. Data from 9,901 children and their parents was included in the analysis. We determined the Body Mass Index (BMI) z-score mean difference as the measurement of the intervention effectiveness and we calculated it as the follow-up ( $\left.T_{1}\right)$ BMI $z$-score mean minus baseline $\left(\mathrm{T}_{0}\right)$ BMI z-score mean. Parents self-reported their employment status at $\mathrm{T}_{0}$ and $\mathrm{T}_{1}$. Children were classified, at both study times $\left(\mathrm{T}_{0}\right.$ and $\mathrm{T}_{1}$ ), as children with employed parents (both parents employed) or as children with unemployed parents (one or both parents unemployed or receiving social assistance). We calculated unadjusted and adjusted multilevel mixed model analyses to evaluate if the employment status at $T_{0}$ and the evolution of the employment status within a two-year period (from $T_{0}$ to $T_{1}$ ) predicted the BMI z-score mean difference among boys and girls. In boys, parental unemployment at $\mathrm{T}_{0}$ and throughout a two-year period $\left(\mathrm{T}_{0}\right.$ to $\left.\mathrm{T}_{1}\right)$ predicted an increase of BMI z-score mean difference when compared to boys with employed parents (unemployment at $\mathrm{T}_{0}$ : adjusted $\beta=0.12 ; p=0.028$; and unemployment from $\mathrm{T}_{0}$ to $\mathrm{T}_{1}$ : adjusted $\beta=0.20 ; p=0.031$ ). We found no difference in the effectiveness of the IDEFICS intervention among girls with unemployed parents at $T_{0}$ and from $T_{0}$ to $T_{1}$ when compared to girls with employed parents (unemployment at $\mathrm{T}_{0}$ : adjusted $\beta=0.04 ; p=0.337$; and unemployment from $\mathrm{T}_{0}$ to $\mathrm{T}_{1}$ : adjusted $\beta=0.10 ; p=$ 0.216 , respectively). Our results suggest that the influence of parental unemployment in the IDEFICS outcome is different for boys and girls. Employment of both parents, which is related to a higher income, could contribute the families to engage healthier eating and physical activity behaviors among boys. Future multilevel interventions should include a combination of community-based and school-based components, as well as family-centered components, specifically on those families with parents out of the labor force, to address specific barriers or vulnerabilities that prevent them from improving behavior and weight status.

\section{Acknowledgements}

IDEFICS Study was funded by European Commission within the Sixth RTD Framework Programme Contract No.016181 (FOOD). AM was financially supported by the Institute of Health Carlos III, Spain (grant\#IFI18/00016).

\section{Conflict of Interest}

The authors of this communication declare that they have no conflicts of interest. 